\title{
A focus on rare and complex kidney diseases
}

\section{Dario Roccatello ${ }^{1}$}

Published online: 15 February 2020

(c) Italian Society of Nephrology 2020
Rare kidney diseases encompass several different conditions, most of which are inherited. Although individual rare kidney diseases raise specific issues, as a group these rare diseases can present overlapping challenges in diagnosis and treatment. These challenges include small numbers of affected patients, unidentified causes of disease, lack of biomarkers for monitoring disease progression, and need for complex care.

The definition of rare diseases varies across the world. In Europe, a disease or disorder is defined as rare when the prevalence is $<1$ in 2000 individuals, whereas in the USA, the designation of a rare disorder applies when $<200,000$ Americans are affected.

Rare kidney diseases include more than 150 different conditions. The majority are inherited, while others, such as the primary glomerulonephritides, have complex etiologies. For inherited kidney disorders, diagnosis, management, and treatment are complex, especially when they are associated with multisystem complications. Patients often waste years visiting multiple health care providers before receiving an accurate diagnosis. Advances in kidney replacement therapy and its increased access allow prolonged patient survival, yet often with a poor quality of life. In particular, children born with severe congenital nephropathies face a high likelihood of altered physical, cognitive, and psychosocial development.

Therapeutic advances in rare kidney diseases have been hindered by several factors, including unidentified genetic defects, lack of biomarkers to monitor disease progression, heterogeneous clinical phenotypes, and outdated diagnostic classifications that do not reflect the underlying pathophysiological mechanisms. Many initiatives have focused on rare kidney diseases, and regulations have been created to promote the development of therapies, organize optimal health care

Dario Roccatello

dario.roccatello@unito.it

1 CMID-Nephrology and Dialysis Unit (ERK-Net Member), Center of Research of Nephrology, Rheumatology, and Rare Diseases, Interregional Coordinating Center of the Network of Rare Diseases of Piedmont and Aosta Valley, G. Bosco Hospital and University of Turin, Turin, Italy systems, and encourage research. Patient organizations have also taken a leading role in these matters. Together, technological advances and organized advocacy have helped enhance our understanding of the clinical, genetic, and mechanistic issues in many inherited kidney disorders.

Among others, it is worth noting that in March, 2017, the European Reference Network for rare kidney diseases (www. erknet.org) was set up together with 23 other rare disease networks during a conference in Vilnius, LT. The European Commission thus finally recognized the eminent importance of improving the clinical management of patients suffering from rare diseases. Studies have shown that currently, establishing diagnosis of these diseases is delayed on average by 5 years thereby creating considerable uncertainty for patients and their families. Furthermore, there is an enormous lack of effective therapies compared with more prevalent diseases.

This new Topical Collection of the Journal of Nephrology on rare and complex kidney diseases aims to provide an updated overview on the incredible scope of research of this thriving kidney research field, thus attesting to the significant progress that has been made towards improving the medical management of patients suffering from these genetic, autoimmune/autoinflammatory and metabolic diseases of the kidney.

The Topical Collection will involve diagnostic challenges, management of kidney function decline and progression of chronic kidney disease, translation of advances in research to clinical care, and provision of practical and integrated patient support. Here, we will collect cutting-edge original studies and expert reviews on selected genetic and autoimmune diseases that nicely illustrate both recent research progress at this fast-moving experimental nephrology frontier and the many open questions that we are still facing.

\section{Compliance with ethical standards}

Conflict of interest The author declares that there is no conflict of interest.

Publisher's Note Springer Nature remains neutral with regard to jurisdictional claims in published maps and institutional affiliations. 